\title{
Intelligent Parking Based on Geolocation Using IoT
}

\author{
Fathima Huda Sirur, Harini P, Javeriya Sajjad, Noor Fathima F, Usha Rani J* \\ Department of Computer Science and Engineering, GSSS Institute of Engineering and \\ Technology for Women, Mysuru, India
}

DOI: https://doi.org/10.21467/proceedings.1.24

* Corresponding author email: usharani@gsss.edu.in

\begin{abstract}
The project "Intelligent parking based on geolocations using Io'T" is aimed to guide people around the city and make them aware of the parking restrictions using Internet of Things. Every person who owns a vehicle has been ticketed at least once due to parking issues. Nowadays every vehicle equipped with GPS technology and smart gadgets, but it miserably fails to avoid parking issues. Thus, we are creating a cloud enabled backend service for the city traffic controller to create Geolocation using polygons which will be sending true or false whether the point asked for resides within the polygon.
\end{abstract}

Index Terms- Arduino MEGA; Buzzer; Geolocations; Mongo DB; PHP; Sensor.

\section{INTRODUCTION}

It's a problem in big cities to get parking: searching for a vacant parking space takes a long time. Car parks are often full during term time though many vehicles do not have parking permits. There is no deterrent to unauthorized parking in unmarked or reserved bays. The car park management system will enable us to make more efficient use of available spaces by controlling vehicle access at key entrances. It will also provide a means of enforcing parking policy, by issuing warning notices and fines to people parking illegally. To build a smart parking system it is necessary to have some sensors that can be applied to a system. We know the parking can be an issue for people. That's why we are planning to introduce a park management system to more effectively control parking and enhance the environment for everyone. Every person who owns a vehicle has been ticketed at least once due to parking issues. Nowadays every vehicle equipped with GPS technology and smart gadgets, but it miserably fails to avoid parking issues. Thus, we are creating a cloud enabled backend service for the city traffic controller to create Geolocation using polygons which will be sending true or false whether the point asked for resides within the polygon. The embedded device inside the vehicle is constantly sending its data during tracking, which we are utilizing to query a geospatial command when the speed decreases to a stationary point and reply back whether it is safe to park or not.

(C) 2018 Copyright held by the author(s). Published by AIJR Publisher in Proceedings of the $3^{\text {rd }}$ National Conference on Image Processing, Computing, Communication, Networking and Data Analytics (NCICCNDA 2018), April 28, 2018.

This is an open access article under Creative Commons Attribution-NonCommercial 4.0 International (CC BY-NC 4.0) license, which permits any non-commercial use, distribution, adaptation, and reproduction in any medium, as long as the original work is properly cited. ISBN: 978-81-936820-0-5 
Intelligent Parking Based on Geolocation Using IoT

2 LITERATURE SURVEY

Literature survey is an important step in software development process. In various referred papers, authors have discussed about different factors that affect the parking process, methods used to enhance parking system, techniques used in tracking and parking. The papers suggest that various protocols, techniques that can be used to overcome drawbacks of the existing system.

\subsection{A Cloud-Based Intelligent Car Parking Services for Smart Cities}

This paper presents the generic concept of using cloud-based intelligent car parking services in smart cities, as an important application deployed on the Internet of Things (IoT) paradigm. The corresponding IoT sub-system includes sensor layer, communication layer, and application layer. A high-level view of the system architecture is outlined. To demonstrate the provision of car parking services with the proposed platform, a cloud-based intelligent car parking system for use within a University campus is described along with details ofdesign and implementation.

\subsection{MQTT protocol}

Message queuing telemetric transport is a publish subscribe based messaging protocol. TCP and UDP protocols are used for IOT appliances. There are various other protocols XMPP, DDS, AMQP used to handle various issues. It represents M2M protocol. MQTT protocol has several benefits like increased scalability, reduction in bandwidth consumption, efficient distribution of information. This protocol connects devices and network with middleware and applications. The default port where it works is on TCP/IP port. The various types of MQTT are hivemq, pahoMQTT. It is most widely used connection protocol for M2M and IOT [8][20].

\subsection{CVMS: Cloud Based Vehicle Monitoring System in VANETs:}

Integration of Cloud computing with VANET is supposed to be the next big thing because of its scalability and reliability. In vehicular ad hoc networks (VANETs) the network services and applications (e.g., safety messages, vehicle navigation data) require an exchange of vehicle and event location information. This paper proposes a new VANET-Cloud integrated service called CVMS: (Cloud Based Vehicle Monitoring System in VANETs) as service in which, vehicles moving on the road serve as witnesses of designated events, capture the real time video or photo of specific location, route or a deadly accident. A group of vehicles with mounted on-board navigation units collaborate to form the vehicular cloud and sends the real time data to central cloud using roadside cloud. The proposed real time cloud-based video capture system has been experimented with various scenarios of video based road services. The algorithm for efficient lane changing, navigation data transferring from vehicular cloud to the central cloud in a real time environment has been implemented on a simulator of an onboard camera based embedded system. The goal of this service is to recognize and track faulty vehicle, emergency vehicles, video and photo capturing of any event, route or location. The 
presented work demonstrates the potential of our proposed system for enhancing and diversifying real-time video services in road environments.

\section{PROPOSED METHOD}

We are creating a cloud enabled backend service for the city traffic controller to create Geolocation using polygons which will sending true or false whether the point asked for resides within the polygon. In our project we are using five components GPS, MCU, Sensor, Buzzer, Sim800. GTO P019 is the GPS used. GPS is used for tracking locations. The microcontroller used is Arduino Mega. Arduino consists of printed circuit board and a piece of software or IDE(Integrated development environment) that runs on your computer to write and upload computer code to the physical board.The sensor is used in the form of a switch, it triggers when vehicles goes from on state to off state.Buzzer sends alert messages like whether the vehicle is parked at a parking zone or not. Sim800 is used to get latitude and longitude.

\section{DESIGN}

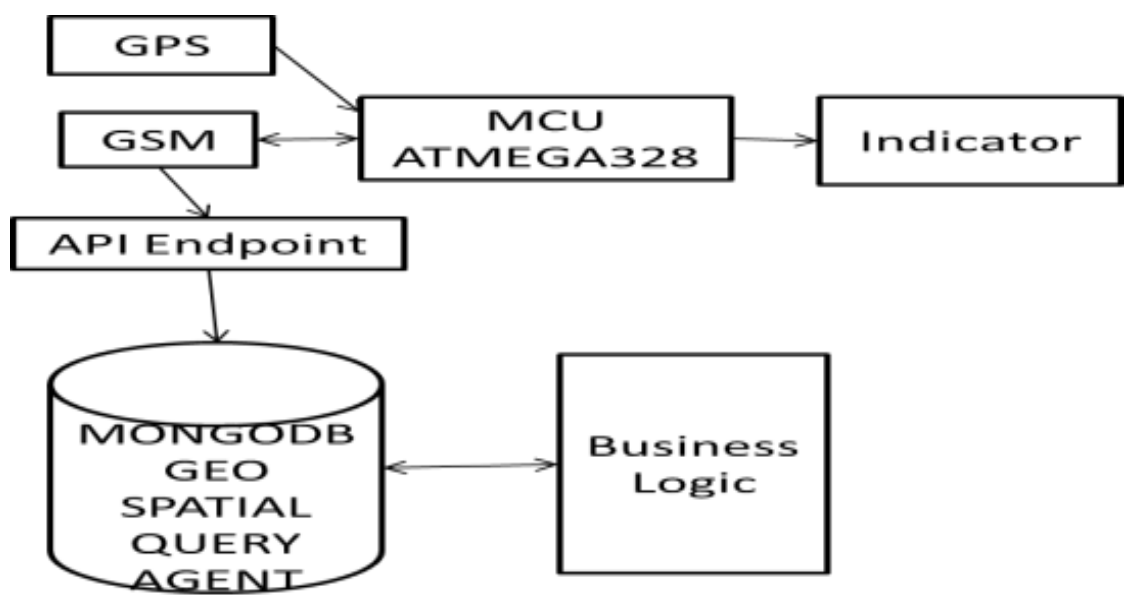

fig: Block Diagram

The main components are Server, Client and Admin Front End. For the Admin to work we need a web server. Mongo DB is used to run the spatial queries and it is also easy to work with and supports multiple arrays. Business logic holds the logic that is coded. Arduino Mega is chosen as GSM and GPS works as UART port. JSON is a data interchange format which is easy for machines to parse and generate.

Proceedings of the $3^{\text {rd }}$ National Conference on Image Processing, Computing, Communication, Networking and Data Analytics (NCICCNDA 2018) 


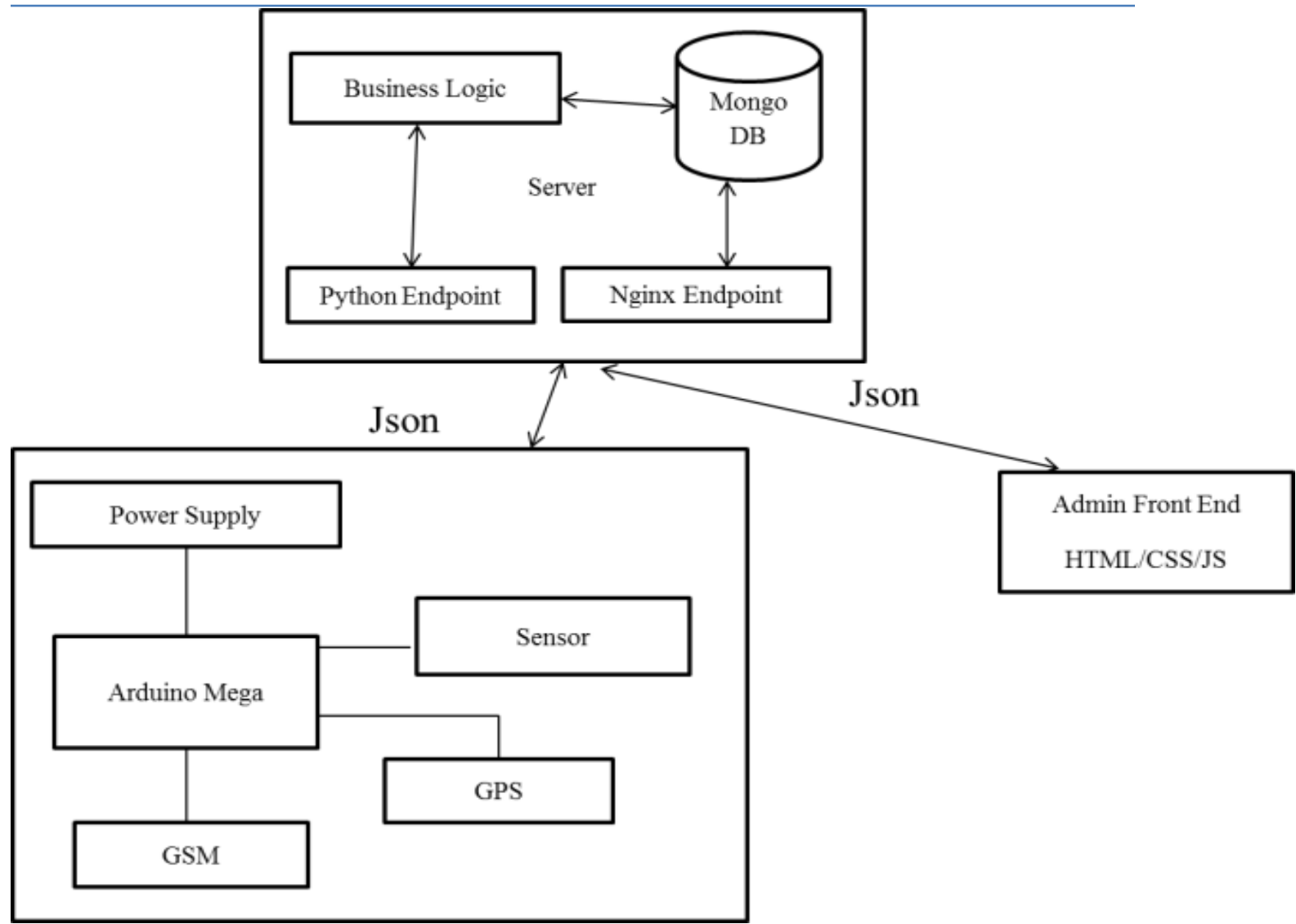

fig: System Architecture

\section{CONCLUSION}

The project "Intelligent parking based on geolocations using Io T" is aimed to guide people around the city and make them aware of the parking restrictions using Internet of Things. The park management system will enable us to make more efficient use of available spaces by controlling vehicle access at key entrances. It will also provide a means of enforcing parking policy, by issuing warning notices and fines to people parking illegally. Our project will check whether a vehicle is in parking or no parking area. If it is in the no parking buzzer will beep and a notification will be sent to the admin.

\section{REFERENCES}

[1] Abhirup Khanna, Rishi Anand "IoT Based Smart Car Parking system" international conference on internet of things applications, IEEE conference publication, pp(260-270), Pune, India, 22Jan-24Jan, 2016.

[2] Conference on Intelligent Sensors, Sensor Networks and Information Processing(ISSNIP) (pp. 1-6).2015, April.

[3] D. Shoup, "Cruising for parking”, ACCESS, No. 30, Spring 2007, pp. 16-22, 2007.

[4] S.D. Galligan. Ibm global parking survey: Drivers share worldwide parking woes. [Online]. Available: https://www03.ibm.com/press/us/en/pressrelease/35515.wss.

[5] Research and Innovative technology administration (RITA), (USDOT) US Department of transportation 1200 New Jersey Avenue. 
Sirur et al., NCICCNDA 2018, AIJR Proceedings 1, pp.143-147, 2018

[6] ERTICO Intelligent Transport Systems and Services stakeholders in Europe: http://www.ertico.com/ertico-its Europe, last accessed on June $10^{\text {th }}, 2014$.

[7] C. M. Rudin-Brown, "Intelligent in-vehicle intelligent transport systems: Limiting behavioral adaptation through adaptive design, "Intelligent Transport Systems, IET, vol. 4,PP. 252-261, 2010.

[8] V. P. Bilodeau, "Intelligent parking technology adoption, "University of Southern Queensland, 2010.

[9] Saif Al-Sultan, Moath M. Al-Doori, Ali H. Al-Bayatti,HussienZedan, "A comprehensive survey on vehicular Ad Hoc network" Journal of Network and Computer Applications, Volume 37, January 2014, Pages 380-392.

[10] Rongxing, L., Xiaodong, L, Zhu, H. and Xuemin, S., "An intelligent secure and privacy-preserving parking scheme through vehicular communications”, Vehicular Technology, IEEE Transactions on, 2010, Vol. 59,pp.2772-278.

Proceedings of the $3^{\text {rd }}$ National Conference on Image Processing, Computing, Communication, Networking and Data Analytics (NCICCNDA 2018) 